\title{
Who Owns the Baby in NICU?
}

\author{
Deepak Chawla ${ }^{1}$ (D)
}

Received: 5 June 2021 / Accepted: 14 June 2021 / Published online: 24 June 2021

(c) Dr. K C Chaudhuri Foundation 2021

What do we mean when we say that the NICU care has become family-centered? Does training a mother and asking her to partially take over some of the nursing responsibilities make it family-centered care (FCC)? Or is it just a method to meet the challenge of perennially adverse nurse-baby ratio in most NICUs of low- and middle-income countries? To answer these questions, as healthcare providers (HCP), we must first examine the relationship between health system and family. Most commonly, when a sick or preterm neonate is admitted to NICU, the healthcare team not only provides the medical care, but also becomes a temporary guardian (the "paternalistic model" of healthcare) and starts to control the access to the baby [1]. The baby is secluded from the family and the NICU team decides when, how, and who from the family can visit the baby. Many units including public sector special newborn care units (SNCUs) do not allow mothers to come inside the unit at all. Mothers may be asked to come every few hours to an adjoining room where baby, if stable enough, would be handed over to be fed and then taken back. Some units may allow mothers inside NICU/SNCU, but timings are limited to suit the staffing pattern and healthcare activities. A smaller number of units allow $24 X 7$ access to mothers and a still smaller number allow mothers to do some care activities like skin-to-skin contact, helping in gavage feeding, and changing nappies. However, FCC must encompass a more holistic model of relationship with the family, in which, mothers (and other family members) are seen not only as suppliers of warmth and milk but as true partners or even owners of the care of their babies.

World Health Organization has outlined the standards of care for sick and preterm neonates to improve the quality of care [2]. The fourth standard states:

"communication with small and sick newborns and their families is effective, with meaningful participa-

Deepak Chawla

drdeepakchawla@gmail.com

1 Department of Neonatology, Government Medical College Hospital, Chandigarh 160 030, India tion, and responds to their needs and preferences, and parental involvement is encouraged and supported throughout the care pathway."

The care standard includes keeping the family informed so that they are able to understand the diagnosis and treatment plan of the baby, accurate information sharing across the multidisciplinary team caring for the baby, making family able to participate in the care of neonate including providing kangaroo mother care, in decision-making, giving informed consent, making treatment choices, and ensuring continuum of care after discharge. American College of Critical Care Medicine defines FCC as "an approach to health care that is respectful of and responsive to individual families' needs and values" [3]. Operational guidelines of the Government of India defines family-participatory care as the one which involves families of sick and preterm neonates as partners in caregiving and decision-making [4]. Roué et al., in their eight principles of patient-centered care and FCC, include as first principle the "free $24 \mathrm{~h}$ a day parental access without any limitations due to staff shifts or medical rounds" [5]. Other seven principles include psychosocial support to parents, pain management, supportive environment, postural support, skin-to-skin contact, breast-feeding and lactation support, and sleep protection. Of these eight, six principles are supported by evidence from systematic reviews.

A recent multicentric study shows improved neonatal survival when mothers and sick neonates are provided health care together in a mother-newborn care unit (MNCU), thus enabling early initiation of SSC and greater involvement of families [6]. However, FCC implementation is easier said than done. Barriers to implementation include space and infrastructural constraints, HCP's existing beliefs and attitudes, and lack of training. In the current issue of the journal, Sivanandan et al. present a study demonstrating successful use of quality-improvement approach to implement the FCC in a level-2 unit [7]. FCC practices included expanded visitation hours, parental education through audiovisual aids and capacity building through training and peer support. The 
authors showed that quality-improvement cycles resulted in greater proportion of mothers participating in the care of their stable babies. With literature showing improved health outcomes with FCC, implementation research is needed to facilitate widespread adoption. However, FCC needs not only adequate space and human resources to support the family, but also a change in the way we, as neonatal care providers, view our relationship with parents. The baby may be admitted with us, but is owned by parents, not us!

\section{Declarations}

Conflict of Interest None.

\section{References}

1. Emanuel EJ, Emanuel LL. Four models of the physician-patient relationship. JAMA. 1992;267:2221-6.
2. World Health Organization. Standards for improving quality of care for small and sick newborns in health facilities. Geneva. 2020.

3. Davidson JE, Aslakson RA, Long AC, et al. Guidelines for familycentered care in the neonatal, pediatric, and adult ICU. Crit Care Med. 2017;45:103-28.

4. Child Health Division, Ministry of Health and Family Welfare, Government of India. Family participatory care for improving newborn health. Operational guidelines for planning and implementation. New Delhi; 2017.

5. Roué JM, Kuhn P, Lopez Maestro M, et al. Eight principles for patient-centred and family-centred care for newborns in the neonatal intensive care unit. Arch Dis Child Fetal Neonatal Ed. 2017;102:F364-8.

6. WHO Immediate KMC Study Group. Immediate "Kangaroo Mother Care" and Survival of Infants with Low Birth Weight. N Engl J Med. 2021;384:2028-38.

7. Sivanandan S, Bethou A, Ramanujam SS, et al. Implementing familycentered care in the neonatal intensive care unit - a quality improvement initiative. In: Indian J Pediatr. 2020. https:// doi.org/10.1007/s12098-020-03566-8.

Publisher's Note Springer Nature remains neutral with regard to jurisdictional claims in published maps and institutional affiliations. 